\title{
CARACTERIZAÇÃO DO ESTADO DE HUMOR DE MULHERES FISICAMENTE ATIVAS E SUA RELAÇÃO COM A IDADE CRONOLÓGICA E COM O ÍNDICE DE MASSA CORPORAL
}

\author{
CHARACTERIZATION OF THE MOOD STATE OF PHYSICALLY ACTIVE WOMEN \\ AND ITS RELATION TO CHRONOLOGICAL AGE AND BODY MASS INDEX
}

\author{
Josivaldo de Souza Lima ${ }^{a^{*}}$, Sandra Marcela Mahecha Matsudo ${ }^{\text {*** }}$, Timóteo Leandro de Araújo ${ }^{c^{*}}$, \\ Victor Keihan Rodrigues Matsudo ${ }^{\mathrm{d}^{*}}$ \\ ajosivaldoesporte@gmail.com, bsandra.mahecha@umayor.cl, ctimoteo@celafiscs.org.br, ${ }^{\mathrm{d}}$ matsudo@celafiscs.org.br \\ *Centro de Estudos do Laboratório de Aptidão Física - São Caetano do Sul (SP), Brasil \\ **Facultad de Ciencias - Universidad Mayor, Santiago de Chile \\ **Grupo de Investigación Movimiento Humano y Salud (GIMHUS), Facultad de Ciencias de la Salud, \\ Universidad de San Buenaventura, Cartagena, Colombia
}

\section{RESUMO}

Data de recebimento do artigo: 26/10/2015 Data de aceite do artigo: 04/03/2016

Objetivo: Associar e comparar o estado de humor com o peso corporal, índice de massa corporal (IMC) e a idade em mulheres participantes de um programa de atividades físicas. Métodos: Estudo de corte transversal com amostra constituída por 435 mulheres com idade média de 68,3 7,9 anos, participantes de um programa de atividades físicas no município de São Caetano do Sul (SP). A amostra é parte do Projeto Longitudinal de Envelhecimento e Aptidão Física de São Caetano do Sul. O tempo de participaçáo da amostra no programa de atividades físicas foi de aproximadamente $8,8 \pm 8,5$ anos, e todas as participantes atendiam a recomendação de atividade física - caminhada por 30 minutos, pelo menos cinco dias na semana - analisado pela versão curta do Questionário Internacional de Atividade Física (International Physical Activity Questionnaire). O IMC foi utilizado seguindo a classificaçấo na Organizaçâo Mundial de Saúde. Subdividimos a amostra por década etária, a seguir: 50 a 59, 60 a 69, e >70. O perfil de estado de humor (Profile of Mood States - POMS) foi avaliado pelo questionário original com 65 itens. Foi feita a análise de comparação do POMS e o IMC com o teste de Kruskal Wallis e associação realizada pelo odds ratio (OR). Resultados: Independentemente da década etária, a maioria da amostra apresentou excesso de peso, variando de $43 \%$ a $50 \%$ ( $<<0,05)$. No perfil de humor foi encontrado um maior escore no grupo classificado como obeso $(16,0)$ e um menor escore no grupo classificado como eutrófico $(8,7)$. O teste de Kruskal Wallis identificou diferenças estatísticas no escore total do POMS $(21,1 \pm 23,1)$ apenas no grupo etário 50-59 anos com excesso de peso, apresentando maior escore em relaçáo ao grupo eutrófico $(3,0 \pm 27,9)$, mas não ao grupo obeso $(22,3 \pm 34,3)$. O IMC eutrófico demonstrou estar associado positivamente com o grupo com melhor resultado na pontuaçáo do POMS (OR: 0,93; intervalo de confiança de $95 \%=0,89$ a 0,97$)$. Conclusão: $\mathrm{Na}$ amostra encontramos relação entre maior índice de massa corporal e alteraçóes negativas no escore do POMS em mulheres ativas. Além disso, foi possível verificar um efeito protetor contra as alteraçóes no escore do POMS no grupo abaixo do percentil 50 do IMC.

Palavras-chave: Humor; envelhecimento; atividade física; índice de massa corporal.

\section{ABSTRACT}

Objectives: To associate and compare the mood state to body weight, body mass index (BMI), and age in women who participate in a physical activity program. Methods: Cross-sectional study with a sample of 435 women with an average age of $68.3 \pm 7.9$ years old, participants in a program of physical activities in São Caetano do Sul, Brazil. The sample is part of the Longitudinal Project of Aging and Physical Fitness in São Caetano do Sul. The sample participation time in the program was approximately $8.8 \pm 8.5$ years and all participants attended to the recommendation of physical activity - walk for 30 minutes, at least five days a week - analyzed by the short version of the International Physical Activity Questionnaire. The BMI was used following the classification of the World Health Organization. The sample was subdivided by age decade: 50-59, 60-69, and $>70$. The Profile of Mood States (POMS) was evaluated through the original questionnaire with 65 items. A comparison analysis was performed between POMS and BMI through the Kruskal Wallis test and association by odds ratio (OR). Results: Regardless of age decade, most of the 
sample presented overweight, varying from 43\% to 50\% ( $<<0.05)$. A higher score was found in POMS in the group classified as obese (16.0) and a lower score in the group classified as eutrophic (8.7). The Kruskal Wallis test identified statistical differences in POMS total score $(21.1 \pm 23.1)$ only in the overweight 50-59 years old age group, with a higher score in comparison with the

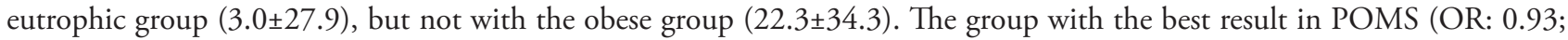
confidence interval of $95 \%=0.89$ to 0.97 ) was positively associated with eutrophic BMI. Conclusions: A relation between higher body mass index and negative changes in POMS score in active women was found in the sample. In addition, a protective effect against changes in POMS score in the group below the 50th percentile of BMI was observed.

Keywords: Mood; aging; physical activity; body mass index.

\section{Introdução}

A manutenção da capacidade física e funcional com a prática regular de exercício físico tem sido recomendada globalmente como uma das medidas importantes para o envelhecimento saudável ${ }^{1,2}$.

Além dos benefícios que proporciona à saúde física, a prática da atividade física também se relaciona positivamente como fator de proteção contra enfermidades crônicas não transmissíveis e doenças psicológicas ${ }^{3-5}$.

Durante o processo de envelhecimento, nos tornamos mais suscetíveis aos distúrbios tanto biológicos quanto psicossociais ${ }^{6}$. Características das mudanças psicossociais se baseiam nas alteraçóes do comportamental, levando ao aumento do estresse mental crônico e a alteraçóes do humor, como demonstrado em outros estudos ${ }^{7-9}$. A alternativa sugerida, além do tratamento medicamentoso, é a terapia com a prática de exercícios físicos, que tem despertado interesse da comunidade científica por seus efeitos promissores na saúde e na sensação de bem-estar relatada por pacientes com enfermidades psicossociais ${ }^{10}$.

Encontramos na literatura que muito tem sido discutido sobre as diversas formas de promover saúde com a prática de atividade física e exercício, no sentido de encontrar a frequência, o modo e intensidade, principalmente na populaçáo com idade avançada ${ }^{11}$.

Entretanto, evidências indicam que um estilo de vida saudável, levando em consideração tanto aspectos físicos quanto mentais, são mais favoráveis à proteção de futuras doenças psicossociais ${ }^{12,13}$.

Nosso estudo enfatiza a análise de alguns dos fatores que a literatura reconhece como promotoras de um estilo de vida saudável, que são a prática de atividades físicas e exercícios físicos e a manutenção do peso corporal, a fim de caracterizar o perfil de estado de humor e sua relaçáo com as diferentes décadas etárias de mulheres praticantes de atividades físicas.

O conhecimento dessa relação poderá auxiliar na elaboração de estratégias e incentivo para um maior aporte na promoção de atividades físicas e exercício na populaçáo adulta que não atende a um dos critérios dentre os diversos existentes para um estilo de vida saudável, que é a atividade física e o exercício físico.

\section{Métodos}

Para este estudo, utilizamos uma base de dados de 1997 a 2011 do Projeto Longitudinal de Envelhecimento e Aptidão Física de São Caetano do Sul. Esse projeto tem como objetivo analisar o impacto do envelhecimento nas variáveis de aptidão física, capacidade funcional, aspectos psicossociais, estado nutricional e nível de atividade física em adultos e idosos praticantes de atividade física e exercícios físicos. O projeto é coordenado, desde 1997, pelo Centro de Estudos do Laboratório de Aptidão Física de São Caetano do Sul. O programa é oferecido pela Prefeitura Municipal de São Caetano do Sul, em São Paulo, e é orientado por um profissional de educação física do Centro Integrado de Saúde e Educação da Terceira Idade. O programa é realizado três vezes na semana, com duração mínima de 60 minutos por aula, e incluem exercícios aeróbicos, alongamento, flexibilidade, mudança de direção, dança e equilíbrio.

Foram utilizados como critérios de exclusão: estar em tratamento medicamentoso por qualquer doença, principalmente doenças que causem distúrbios psicológicos ou alteraçôes cognitivas; e não ter todos os resultados completos das avaliaçóes, inclusive do questionário POMS.

Os critérios de inclusão foram: ser do sexo feminino; ter idade igual ou acima de 50 anos; ter os dados antropométricos e o questionário perfil de estado de humor completos. Todas as participantes assinaram o termo de consentimento. $\mathrm{O}$ estudo foi aprovado pelo Comitê de Ética e Pesquisa de São Caetano do Sul, sob $n^{\circ} 128 / 2010$.

\section{Padronização adotada na avaliação}

O peso corporal foi medido em balança eletrônica (Filizola) com capacidade para 200 quilogramas e com precisão de 100 gramas. A avaliada foi colocada em pé, de costas para a escala da balança, com afastamento lateral dos pés, corpo ereto e com olhar no plano de Frankfurt. A estatura corporal foi medida no estadiômetro, graduado em centímetros e décimos de centímetros. 
A avaliada foi colocada em posição ortostática, com os pés unidos, com a parte posterior dos calcanhares, cintura pélvica, cintura escapular e regiáo occipital encostadas na base fixa. A medida é realizada em apneia inspiratória e com a cabeça no plano de Frankfurt, paralela ao solo. São feitas três medidas e considerada a média como valor real da estatura. Depois, calculamos o IMC $\left(\mathrm{kg} / \mathrm{m}^{2}\right)$. Foi utilizada a padronizaçáo CELAFISCS de avaliação física e funcional ${ }^{12}$. O IMC foi classificado de acordo com a Organização Mundial da Saúde $(\mathrm{OMS})^{13}$, sendo eutrófico $<24,9 \mathrm{~kg} / \mathrm{m}^{2}$, excesso de peso de 25,0 a $29,9 \mathrm{~kg} / \mathrm{m}^{2}$ e obeso $>30 \mathrm{~kg} / \mathrm{m}^{2}$.

Para determinar o perfil de estado de humor foi utilizado o questionário POMS original, ainda não validado no Brasil, mas utilizado em outros estudos brasileiros $^{14}$, cujo objetivo é mensurar distúrbios psíquicos transitórios flutuantes em indivíduos acima de 18 anos de idade. O questionário é composto por 58 itens, sendo divididos em seis domínios: nove itens para tensão, com intervalo de pontuação mínima de zero e máxima de trinta e seis; quinze itens para depressão, com intervalo de pontuação mínima de zero e máxima de sessenta; doze itens para raiva, com intervalo de pontuação mínima de zero e máxima de quarenta e oito; sete itens para fadiga, com intervalo de pontuação mínima de zero e máxima de vinte e oito; sete itens de confusão, com intervalo de pontuação mínima de zero e máxima de vinte e oito; e oito itens para vigor, com intervalo de pontuação mínima de zero e máxima de trinta e dois. Os primeiros cinco domínios citados são de aspectos negativos; portanto, quanto maior o valor apresentado nesses domínios, pior o perfil de estado de humor, e o único item considerado como positivo é o vigor. Durante o preenchimento, o avaliado responde para cada item: "Como você está se sentindo nas últimas semanas, inclusive hoje?", dando para cada item um valor numérico de: 0 , nunca; 1 , um pouco; 2 , moderadamente; 3 , bastante; e 4, extremamente.

Os questionários foram preenchidos pelas participantes em um tempo que variou de 5 a 10 minutos. Após o preenchimento, realizou-se o cálculo matemático, pela soma de todos os itens considerados negativos e foi subtraído o item considerado positivo para assim determinar o perfil total de humor.

O nível de atividade física foi avaliado utilizando o IPAQ versão $\operatorname{curta}^{15}$. A frequência mínima desse grupo de participantes nas aulas foi de $75 \%$, sendo esse um dos critérios para que se mantivessem inscritas no programa oferecido pelo município. $\mathrm{O}$ tempo médio de prática desse grupo foi de $8,8 \pm 8,5$ anos. As avaliaçóes eram realizadas duas vezes por ano, nos meses de maio e novembro, totalizando, até a data desta publicação, um banco de dados de aproximadamente seis mil avaliaçôes. A base inicial da amostra foi composta por 4.062 indivíduos, sendo incluídas nesta pesquisa apenas 435, o que representava $10,7 \%$ da amostra total. O grupo foi dividido, de acordo com a idade cronológica, em três grupos: 50 a 59, 60 a 69, e $>70$.

\section{Análise estatística}

A análise de Kolmogorov Smirnov foi usada para determinar a normalidade dos dados, a associação foi realizada pelo odds ratio (OR). Para realizar a comparação do perfil de estado de humor com o IMC foi utilizado o teste de Kruskal Wallis seguido de U de Mann-Whitney, para localizar as possíveis diferenças. Os cálculos foram realizados pelo software Statistical Package for the Social Sciences (SPSS), versão 6.0, e o nível de significância adotado foi de $\mathrm{p}<0,05^{16}$.

\section{Resultados}

Do total da amostra que participou da pesquisa, a média de idade foi de $68,3 \pm 7,9$ anos. A maioria da amostra (46\%) apresentou excesso de peso $(25,0 \mathrm{a}$ $\left.29,9 \mathrm{~kg} / \mathrm{m}^{2}\right)$, apenas $28 \%$ foi classificada como eutrófica $\left(<24,9 \mathrm{~kg} / \mathrm{m}^{2}\right)$ e $26 \%$ foi classificada como obesa $\left(>30 \mathrm{~kg} / \mathrm{m}^{2}\right)$.

Em relação à atividade física, $82 \%$ atingiam a recomendação para atividades físicas e $46 \%$ atendiam à recomendação de caminhada cinco vezes na semana por 30 minutos.

A Tabela 1 mostra a distribuição da amostra de acordo com a classificação do IMC em eutrófico, excesso de peso e obeso, com a década etária $(50$ a 59, 60 a 69 e $>70$ anos). Analisados os resultados, não encontramos diferenças estatisticamente significantes no IMC de acordo com a faixa etária.

Tabela 1: Características gerais da amostra de acordo com a década etária e a classificação do índice de massa corporal (IMC).

\begin{tabular}{|cccc|}
$\begin{array}{c}\text { Idade } \\
\text { (anos) }\end{array}$ & Eutrófico & Excesso de Peso & Obeso \\
\hline $50-59$ & n (\%) & n (\%) & n (\%) \\
\hline $60-69$ & $50(27,6)$ & $22(47,7)$ & $16(29,6)$ \\
\hline 70 & $54(27,4)$ & $78(43,0)$ & $53(29,2)$ \\
\hline $\mathrm{p}<0,05$. & & $99(50,2)$ & $45(22,8)$ \\
\hline
\end{tabular}

O grupo com maiores valores de perfil total de humor também apresentaram maior IMC ( $\mathrm{p}<0,05)$, e apenas $27,7 \%$ $\mathrm{da}$ amostra apresentou IMC adequado no grupo etário $>70$ anos de idade. Os resultados do perfil de estado de humor de acordo com o IMC estão apresentados no Gráfico 1. 
Gráfico 1: Perfil do estado de humor de acordo com índice de massa corporal de mulheres adultas praticantes de atividade física.

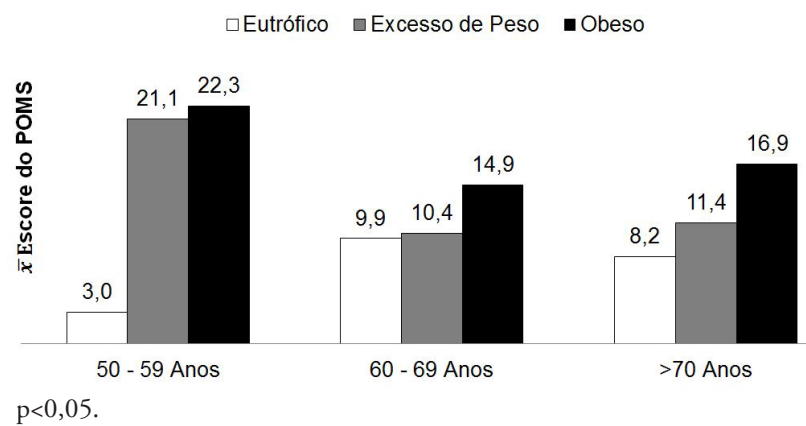

Ao analisar o perfil de estado de humor de acordo com a década etária e segundo a classificação do IMC, foram encontradas diferenças estatísticas no grupo etário 50-59 anos de idade. No item raiva-hostilidade, o grupo excesso de peso apresentou maiores valores $(10,4 \pm 6,0)$ quando comparado ao grupo eutrófico $(6,3 \pm 8,1)$. O item confusão mental apresentou diferenças significantes em todos os grupos de IMC: eutrófico $(0,37 \pm 3,3)$; excesso de peso $(3,6 \pm 4,9)$; e no grupo obeso $(6,2 \pm 5,1)$.

$\mathrm{O}$ mesmo fenômeno náo foi observado de maneira significativa na década etária de 60-69 nos itens do perfil de estado de humor segundo o IMC. Já na década etária $>70$ anos de idade, foram encontradas diferenças significativas entre o item raiva-hostilidade do grupo eutrófico $(6,2 \pm 7,8)$ e o grupo obeso $(8,8 \pm 7,0)$.

Tabela 2: Perfil de estado de humor de acordo com o índice de massa corporal e idade cronológica de mulheres adultas praticantes de atividade física.

\begin{tabular}{|c|c|c|c|c|c|c|}
\hline Estado de Humor & Eutr & fico & Excess & de Peso & $\mathrm{Ob}$ & eso \\
\hline 50 - 59 ANOS & $\bar{x}$ & $\mathbf{s}$ & $\bar{x}$ & $\mathbf{s}$ & $\bar{x}$ & $\mathbf{s}$ \\
\hline Tensão e Ansiedade & 4,3 & 7,2 & 8,8 & 6,5 & 9,5 & 6,5 \\
\hline Depressão & 5,1 & 6,5 & 8,1 & 6,2 & 9,7 & 9,1 \\
\hline Raiva e Hostilidade & 6,3 & 8,1 & $10,4^{\mathrm{a}}$ & 6,0 & 9,7 & 9,6 \\
\hline Vigor & 17,5 & 7,0 & 15,0 & 6,2 & 16,2 & 5,9 \\
\hline Fadiga & 5,2 & 5,8 & 5,2 & 4,8 & 6,3 & 5,5 \\
\hline Confusão Mental & $-0,37$ & 3,3 & $3,6^{a}$ & 4,9 & $6,2^{a}$ & 5,1 \\
\hline Score Total & 3,0 & 27,9 & $21,1^{a}$ & 23,1 & 22,3 & 34,3 \\
\hline $60-69$ ANOS & $\bar{x}$ & $s$ & $\bar{x}$ & $s$ & $\bar{x}$ & $s$ \\
\hline Tensão e Ansiedade & 7,4 & 6,1 & 7,8 & 6,5 & 7,0 & 6,8 \\
\hline Depressão & 6,9 & 6,8 & 6,8 & 7,4 & 8,3 & 8,3 \\
\hline Raiva e Hostilidade & 6,7 & 5,9 & 7,4 & 7,0 & 8,0 & 7,2 \\
\hline Vigor & 17,3 & 6,0 & 17,9 & 5,5 & 15,6 & 5,8 \\
\hline Fadiga & 4,8 & 3,8 & 4,9 & 4,5 & 4,9 & 4,4 \\
\hline Confusão Mental & 1,5 & 3,7 & 1,4 & 4,2 & 2,2 & 4,4 \\
\hline Score Total & 9,9 & 22,8 & 10,4 & 25,6 & 14,9 & 29,0 \\
\hline$>70$ ANOS & $\bar{x}$ & $s$ & $\bar{x}$ & $s$ & $\bar{x}$ & $s$ \\
\hline Tensão e Ansiedade & 6,1 & 6,8 & 6,9 & 6,5 & 8,1 & 6,5 \\
\hline Depressão & 6,7 & 8,8 & 7,1 & 8,7 & 9,0 & 8,8 \\
\hline Raiva e Hostilidade & 6,2 & 7,8 & 7,6 & 7,8 & $8,8^{\mathrm{a}}$ & 7,0 \\
\hline Vigor & 17,0 & 5,0 & 17,4 & 6,2 & 16,9 & 7,1 \\
\hline Fadiga & 4,6 & 5,2 & 5,5 & 4,9 & 5,6 & 5,0 \\
\hline Confusão Mental & 1,5 & 4,6 & 1,7 & 4,4 & 2,2 & 4,5 \\
\hline Score Total & 8,2 & 29,7 & 11,4 & 29,9 & 16,9 & 28,3 \\
\hline
\end{tabular}

Ao comparar o escore total do perfil de estados de humor nos grupos etários de acordo com o IMC foram encontradas diferenças estatisticamente significativas entre o grupo eutrófico de 50-59 anos de idade $(3,0 \pm 27,9)$ e o grupo de excesso de peso $(21,1 \pm 23,1)$. $\mathrm{Na}$ faixa etária de 50-59 anos de idade observamos que o grupo eutrófico $(3,0 \pm 27,9)$ apresentou menor escore total quando comparado com o grupo excesso de peso $(21,1 \pm 23,1) \quad(p<0,05)$, mas não apresentou diferenças estatisticamente significantes quando comparado com o grupo obeso $(22,3 \pm 34,3)$, que apresentou maior escore total. O mesmo não foi observado nos grupos etários de 60-69 e >70 anos de idade.

Gráfico 2: Perfil do estado de humor (ESCORE TOTAL) de acordo com o índice de massa corporal e idade cronológica de mulheres participantes de programa de atividade física.

$\square$ Eutrófico $\square$ Excesso de Peso $\square$ Obeso

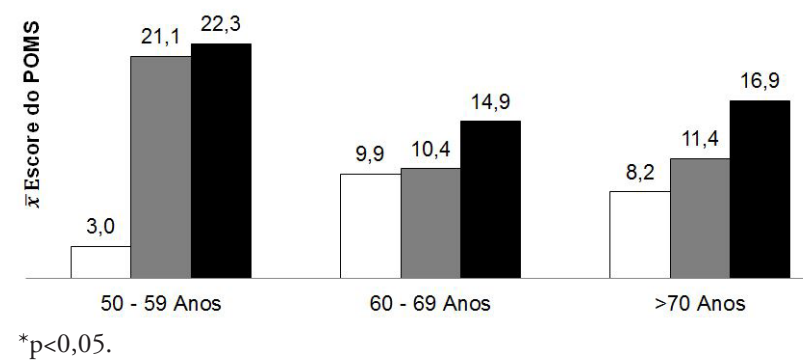

A Tabela 3 mostra os resultados da associação do perfil de estados de humor e índice de massa corporal em mulheres praticantes de atividade física, ajustada pela idade. Foi realizada por meio da dicotomização da variável POMS, dividida pelo percentil 50, sendo a referência o grupo com melhor escore no POMS. As mulheres que apresentavam melhor perfil de estado de humor apresentaram 7\% de chance de ter o IMC adequado.

Tabela 3: Análise ajustada da associação do perfil de estado de humor e índice de massa corporal em mulheres praticantes de atividade física.

\begin{tabular}{cccc|}
$\operatorname{IMC}\left(\mathbf{k g} / \mathbf{m}^{\mathbf{2}}\right)$ & \multicolumn{3}{c|}{ POMS } \\
& $>$ percentil 50 & $<$ percentil 50 \\
& Referência & OR (IC95\%) & p \\
& 1,00 & $0,93(0,89-0,97)$ & 0,003
\end{tabular}

Resultado de regressão logística binária ajustada para idade, com resultados expressos em odds ratio (OR) e intervalos de confiança de 95\% (IC95\%).

\section{Discussão}

O objetivo deste estudo foi associar e comparar o estado de humor com o peso corporal, índice de massa corporal e idade em mulheres participantes de um 
programa de atividade física. A população estudada apresenta $8,8 \pm 8,5$ anos de participação no programa oferecido pelo município e apresenta alta adesão.

Foi encontrado um maior escore de raiva e hostilidade no grupo com idade entre 50-59 anos com excesso de peso, que representa 10,4 do escore. Também observamos pior valor de confusão mental no grupo com excesso de peso e obeso em relação ao eutrófico, 3,6 e 6,2 , respectivamente. Já no escore total, avaliando os seis itens, o pior resultado foi encontrado na década etária de 50-59 anos, sendo o grupo com excesso de peso com o valor de 21,1 do escore total.

Quando analisamos o grupo da década etária entre 60-69 anos, não observamos diferenças em relação ao IMC, tampouco aos itens. Náo necessariamente pode ser considerada a década etária com melhores resultados na avaliação do humor; entretanto, nessa década etária podemos dizer que não importa se o individuo tem 60 ou 69 anos, o seu perfil de humor é igual independente da idade dentro desses limites anteriormente ditos.

Já em relação à avaliação de humor no grupo acima de 70 anos, foi possível observar maior valor no item raiva e hostilidade no grupo considerado obeso em relação ao grupo eutrófico, e não encontramos diferenças no grupo com excesso de peso.

Por fim, resumimos esses achados identificando que na primeira década etária analisada observamos piores valores no grupo excesso de peso e obeso, no grupo da década etária 60-69 não se observou diferenças dentro dos limites predefinidos e no grupo com idade acima de 70 anos apenas se observou piores resultados do POMS no grupo com excesso de peso.

Além da análise comparativa, realizamos o teste de associação binária, no qual calculamos o percentil $<50$ e 50>. Após isso, a associação se mostrou significativa com efeito protetor do IMC OR 0,93 (IC95\%=0,89$0,97)$, mesmo quando ajustado pela idade, ou seja, o percentil $<50$ apresentou proteção contra distúrbios do perfil do humor.

Os achados do estudo apontam diferenças significativas no perfil de estado de humor de acordo com o IMC nas décadas iniciais e finais do processo de envelhecimento. Nos itens raiva-hostilidade e escore total ainda foi possível verificar uma relação direta e significativa entre o IMC e o perfil de estado de humor.

Em um estudo realizado em cidades do mesmo estado desta pesquisa, encontramos efeitos benéficos dos programas de atividades físicas e exercícios físicos na melhora da depressão, bem-estar e capacidade funcional de mulheres acima de 50 anos ${ }^{17}$. Nossa pesquisa não teve como objetivo avaliar o efeito do programa sobre a saúde das praticantes, mas encontramos melhores valores antropométricos dos participantes adultos acima de 50 anos $^{18}$. Os dados encontrados em outro estudo ${ }^{19}$, que analisou o perfil de estados de humor em idosos de um programa estruturado de yoga, apresentaram um aumento significativo no item vigor quando comparado com o grupo controle. Ainda se constatou uma redução nos níveis de tensão, ansiedade e raiva-hostilidade. No presente estudo evidenciamos diferenças nessas mesmas variáveis, sendo significativas na confusão mental e raiva-hostilidade no grupo de mulheres da década de 50 a 59 anos e maior de 70 anos.

Sabe-se que os fatores psicológicos descritos nos itens que compóem o perfil de estado de humor e a alta exigência pessoal em idosos escolarizados estão associados diretamente com queixas de memória, sendo um indicador de dificuldade cognitiva em idosos ${ }^{20,21}$. Pesquisas longitudinais sugerem que essas queixas subjetivas de memória entre idosos sem demência não são apenas secundárias à depressão, mas refletem os status cognitivos ${ }^{22}$.

$\mathrm{O}$ resultado do presente estudo, em relação ao item depressão, não demonstrou diferenças estatisticamente significantes de acordo com o grupo etário. Esse achado corrobora estudo realizado em idosos que praticavam atividades físicas recreativas e que, apesar de não modificarem o item depressão com a prática, tiveram melhora no perfil de estado de humor, concluindo que resta pouca dúvida quanto aos benefícios de exercícios e $\mathrm{da}$ atividade física como estratégia não farmacológica e de alternativa como profilaxia aos agravos mentais, que tem início com alteraçóes do humor decorrentes do envelhecimento $^{23,24}$.

O estudo de Annesi ${ }^{25}$ comparou o efeito de 10 semanas de exercícios aeróbicos e de força muscular no perfil de estado de humor de mulheres de três grupos distintos: grupo controle (idade de 21 a 80 anos), grupo de adultos jovens (idade entre 21 e 45 anos) e grupo de adultos mais velhos (idade entre 55 e 80 anos). Analisando os domínios do POMS no grupo controle, houve aumento não significativo no domínio tensão, depressão, vigor e fadiga. Já o grupo de adultos mais velhos teve diminuição no domínio tensão de 2,3 pontos e no depressão de 1,4 , assim como aumento significativo do vigor, em média 1,1 e fadiga de 1,3 no escore $(\mathrm{p}>0,05)^{25,26}$. Os autores concluíram que atividade física estruturada tem associação com a melhora do perfil de estados de humor em mulheres, independente da idade. Este não é estudo de intervenção e não pode estabelecer relação causa-efeito.

Em outro estudo ${ }^{27}$, que analisou o perfil de estados de humor de indivíduos acima de 60 anos de idade em dois tipos de atividades físicas (caminhada como atividade coletiva e hidroginástica como atividade individual), foi possível verificar que a caminhada foi mais sensível na melhora do escore de humor, de $39 \pm 25$ para $-13 \pm 15$ pós-intervenção $(p<0,05)$. A intervenção foi mais eficaz 
no combate ao estresse e diminuição dos domínios da fadiga, tensão-ansiedade, depressão, raiva-hostilidade e confusão mental. É importante ressaltar que as mulheres em nossa amostra apresentaram valores baixos dos itens considerados negativos para a saúde e isso poderia estar relacionado ao estilo de vida. Nossa amostra é considerada fisicamente ativa, caminham regularmente e algumas realizam hidroginástica, o que poderia estar relacionado ao menor escore. A interaçáo da melhora do humor com a prática da atividade física deve considerar a interaçáo entre os fenômenos fisiológicos e comportamentais.

Ao comparar o perfil de estados de humor e o nível de cortisol 8-hidroxideoxiguanosina (8-Ohdg) com dois grupos de mulheres, grupo controle e grupo praticante de yoga por mais de dois anos, foi observada uma tendência de diminuição dos níveis bioquímicos de cortisol no grupo praticante de yoga, embora as diferenças não fossem significativas. Quanto aos resultados do POMS, o grupo yoga apresentou menor distúrbio total de humor, tensão e ansiedade, raiva-hostilidade e fadiga. Houve também uma tendência a uma pontuação maior no vigor do grupo yoga, não sendo encontradas diferenças significativas nos escores de depressão e confusão $0^{28}$.

Outra questáo importante a ressaltar é que a dor física é uma situação comum em idosos e isso pode interferir negativamente nos resultados do perfil de estado de humor. Um programa de atividades físicas estruturado para mulheres com média de idade de 59 anos com diagnóstico clínico de fibromialgia demonstrou valores baixos no item vigor após determinado tempo de prática. Há evidências, em outra pesquisa, que sugerem que a atividade física em longo prazo pode ser eficaz no aumento do vigor físico relacionado à aptidão física ${ }^{29}$.

Ainda é muito controversa na literatura a duração mínima necessária da prática da atividade física para o efeito nos itens que compóem o perfil de estado de hu$\operatorname{mor}^{30,31}$. No presente estudo, o tempo médio de participação no programa se mostrou um fator positivo na relação IMC e perfil de estado de humor. Porém, evidências mostraram resultados positivos nos aspectos psicossociais de mulheres acima de 50 anos praticantes de atividade física estruturada, encontrando correlação significativa (r: 0,31 e 0,29$)$ entre satisfação com a vida e autoestima nas faixas etárias de 50-59 e 70-79 anos de idade, respectivamente ${ }^{32}$. Esse achado corrobora as associaçóes significativas encontradas em nosso estudo e ainda possibilita verificar um efeito protetor do IMC no perfil de estado de humor. É importante salientar que, nesta pesquisa, o excesso de peso foi utilizado seguindo a padronização da OMS. A hipótese inicial deste estudo era encontrar piores resultados de humor nos indivíduos com IMC elevado, porém essa hipótese não foi encontrada. No entanto, os dados indicaram menor escore de raiva-hostilidade no grupo eutrófico na primeira década etária, 50-59 anos, quando comparado com as demais classificaçóes do IMC. No grupo $>70$ anos de idade também houve menor raiva-hostilidade no grupo eutrófico quando comparado com o obeso.

Ao relacionar a massa corporal e qualidade de vida com saúde de mulheres de meia idade, foi observado que o grupo eutrófico e o grupo excesso de peso mostraram melhores resultados nos aspectos psicológicos e sociais, corroborando a ideia inicial de que a atividade física pode melhorar e/ou manter o peso corporal ideal, que está associado a uma melhor resposta com a qualidade de vida ${ }^{33}$.

Além de melhorar esses aspectos, relacionados à saúde mental, a prática da atividade física melhora o perfil de estado de humor, podendo fazer com que os indivíduos se mantenham por mais tempo matriculados em academias ou em aulas estruturadas, ou seja, maior aderência. Tal fato pode ser explicado pela melhoria das condiçóes psicológicas. Esses indivíduos não apenas obtiveram melhora de condições de saúde de uma forma geral, mas também uma diminuição da tensão, fadiga, confusão, hostilidade, depressão e ainda um alto valor de vigor ${ }^{34}$.

Os aspectos fisiológicos podem causar alterações no comportamento social e emocional dos indivíduos que passam pelo processo de envelhecimento, exercendo influências negativas sobre a qualidade de vida da mulher na pós-menopausa ${ }^{35}$. A transição da menopausa por si não causa alteraçóes no humor de mulheres. $\mathrm{O}$ humor negativo em mulheres pode estar associado com outras variáveis, como condição sociodemográfica, estado de saúde, estresse, queixa pré-menstrual e atitudes em relação ao envelhecimento ${ }^{36}$.

Distúrbios psicossociais são caracterizados por um conjunto de sinais e sintomas, como perda de interesse do prazer em atividades anteriormente significativas, distúrbios de sono, apetite, diminuição do interesse sexual, retardo psicomotor, dificuldade cognitiva, desesperança, diminuição da autoestima e pensamento de morte ou suicida ${ }^{37-39}$. Estes podem ser minimizados com a participação ativa desses indivíduos na sociedade, corroborando nossos achados do grupo de idosos participantes do programa de atividade física e que são fisicamente ativos na comunidade.

Algumas limitaçôes do presente estudo devem ser levadas em consideração, como o viés de seleção e o viés de aferição. A amostra considerada de conveniência foi selecionada de uma base de dados existente de um projeto longitudinal que analisa uma coorte de mulheres envolvidas em um programa de atividade física. Entendemos que a amostra dessa base de dados não possuía alterações cognitivas, físicas ou funcionais que impedissem a vida em comunidade; com isso, os resultados aqui 
apresentados talvez não possam ser aplicados à população que não tenha as mesmas características de estilo de vida. Na interpretação dos resultados, as informaçôes derivadas de questionários de autorrelato podem sofrer influência das funçóes cognitivas do avaliado, levando a um possível viés de aferição.

\section{Conclusão}

Mulheres participantes de um programa estruturado de atividade física apresentaram diferenças de perfil de estado de humor de acordo com a idade e com o IMC nos itens raiva-hostilidade, confusão mental e escore total de humor. Além disso, a associação encontrada se mostrou significativa com efeito protetor do IMC, mesmo quando ajustado pela idade.

Sendo assim, é importante acreditarmos que intervençôes sobre o envelhecimento populacional em nível de atividade física pode proporcionar melhores níveis de saúde física e consequentemente melhor saúde mental.

\section{Agradecimentos}

Os autores agradecem a todos os participantes do projeto, em especial aos centros da terceira idade de Sáo Caetano do Sul, pela continuidade desse projeto longitudinal.

\section{Referências}

1. Anderssen SA, Stromme SB. Physical activity and health: recommendations. Tidsskr Nor Laegeforen. 2001;121(17):2037-41.

2. Sparling PB, Howard BJ, Dunstan DW, Owen N. Recommendations for physical activity in older adults. BMJ. 2015;350:h100.

3. Moreira RM, Teixeira JRB, Boery EN, Boery RNSO, Mota TN. Saúde pública, atividade física e qualidade de vida de idosos: uma reflexão teórica. Saúde e Pesquisa. 2013;6(2):331-7.

4. Garcia MAA, Rodrigues MG, Borega RS. O envelhecimento e a saúde. Rev de Ciênc Méd (Campinas). 2012;11(3):221-31.

5. Calomeni MR, Silva VF, Ribeiro LHB, Cavalcante J, Siza MAF. Exercício físico e plasticidade neurogênica: benefícios relacionados às funçôes mentais do idoso. Biol \& Saúde (Campos dos Goytacazes). 2012;2(6):35-43.

6. Fonseca RP, Trentini CM, Valli F, Silva RAN. Representaçóes do envelhecimento em agentes comunitários da saúde e profissionais da enfermagem comunitária: aspectos psicológicos do processo saúde-doença. Cien Saude Colet. 2008;13(4):1275-84.
7. McEwen BS. Cérebro: o órgão central do estresse e da adaptação ao longo da vida. 2013 [acesso em 4 jul 2016]. Disponível em: http://bit.ly/29tKx79

8. Pinto FNFR, Barham EJ. Habilidades sociais e estratégias de enfrentamento de estresse: relaçáo com indicadores de bem-estar psicológico em cuidadores de idosos de alta dependência. Rev Bras Geriatr Gerontol. 2014;17(3):525-39.

9. Passos J, Sequeira C, Fernandes L. Nursing focuses in older people with mental disorders. Referência. 2014;4(2):81-91.

10. Antunes HKM, Santos RF, Cassilhas R, Santos RVT, Bueno OFA, Mello MT. Exercício físico e função cognitiva: uma revisão. Rev Bras Med Esporte. 2006;12(2):108-14.

11. Miranda REEPC, Mello MT, Antunes HKM. Exercício físico, humor e bem-estar: consideraçóes sobre a prescrição da alta intensidade de exercício. Rev Psicol Saúde (Campo Grande). 2011;3(2):46-54.

12. Gobbi S, Corazza DI, Costa JLR, Ueno DT, Gobbi LTB. Atividade física e saúde no envelhecimento: a experiência do Programa de Atividade Física para a Terceira Idade (PROFIT). In: Rosa TEC, Barroso AES, Louvison MCP, organizadores. Velhices: experiências e desafios nas políticas do envelhecimento ativo. São Paulo: Instituto de Saúde; 2013. p. 283.

13. Carvalho J. Pode o exercício físico ser um bom medicamento para o envelhecimento saudável? Acta Farmacêutica Portuguesa. 2014;3(2):123-30.

14. Gabriel CB, Santos L, Salles PG. Efeitos agudos da atividade física sobre o estado de humor de indivíduos da $3^{\mathrm{a}}$ idade. Revista Saúde Física \& Mental. 2013;2(1):11-21.

15. Matsudo S, Araújo T, Matsudo V, Andrade D, Andrade E, Oliveira LC, et al. Questionário internacional de atividade física (IPAQ): estudo de validade e reprodutibilidade no Brasil. Rev Bras Ativ Fís Saúde. 2001;6(2):5-18.

16. Norusis MJ. SPSS for Windows Advanced Statistics Release 6.0. 6a ed. Engleewod Cliffs: Prentice Hall; 1993.

17. Silva Filho JN, Zamaro M, Ferreira RA. Avaliação de dois programas de atividades físicas no desempenho de mulheres acima de cinquenta anos. Lecturas: Educación Física y Deportes [Internet]. 2013 [acesso em 4 jul 2016];18(187). Disponível em: http://bit.ly/29e3ahg

18. Oliveira DC, Neri AL, D’Elboux MJ. Variáveis relacionadas à expectativa de suporte para o cuidado de idosos residentes na comunidade. Rev Lat-Am Enfermagem. 2013;21(3):742-9.

19. Yoshihara K, Hiramoto T, Sudo N, Kubo C. Profile of mood states and stress-related biochemical indices in long-term yoga practitioners. Biopsychosoc Med. 2011;5(1):1-7.

20. Mewton L, Sachdev P, Anderson T, Sunderland M, Andrews G. Demographic, clinical, and lifestyle correlates of subjective memory complaints in the Australian population. Am J Geriatr Psychiatry. 2014;22(11):1222-32.

21. Wang PN, Liao SQ, Liu RS, Liu CY, Chao HT, Lu SR, et al. Effects of estrogen on cognition, mood, and cerebral blood flow in AD: a controlled study. Neurology. 2000. 13;54(11):2061-6. 
22. Treves TA, Verchovsky R, Klimovitzky S, Korczyn AD. Incidence of dementia in patients with subjective memory complaints. Int Psychogeriatr. 2005;17(2):265-73.

23. Cheik NC, Reis IT, Heredia RAG, Ventura ML, Tufik S, Antunes HKM, et al. Efeitos do exercício físico e da atividade física na depressáo e ansiedade em indivíduos idosos. $\mathrm{R}$ Bras Ci e Mov. 2003;11(3):45-52.

24. Minghelli B, Tomé B, Nunes C, Neves A, Simões C. Comparação dos níveis de ansiedade e depressão entre idosos ativos e sedentários. Rev Psiquiatr Clín. 2013;40(2):71-6.

25. Annesi JJ, Whitaker AC. Weight loss and psychologic gain in obese women-participants in a supported exercise intervention. Perm J. 2008;12(3):36-45.

26. Harris AH, Cronkite R, Moos R. Physical activity, exercise coping, and depression in a 10-year cohort study of depressed patients. J Affect Disord. 2006;93(1-3):79-85.

27. Sabino IG, Zaniqueli D, Lignani AM, Carletti L. Os efeitos da prática de exercícios físicos individuais e coletivos sobre o estado de humor de mulheres idosas. Anais do $16^{\circ}$ Congresso Brasileiro de Ciências do Esporte e III Congresso Internacional de Ciências do Esporte; 2009 set. 20-25; Salvador, Brasil. 2009.

28. Dolde EJ. The Effects of Yoga and Aerobic Exercise on Concentration and Feeling-States. Bristol. Honors Thesis Roger Williams University; 2011.

29. Brandt R, Fonseca ABP, Oliveira LGA, Steffens RAK, Viana MS, Andrade A. Perfil de humor de mulheres com fibromialgia. J Bras Psiquiatr. 2011;60(3):216-20.

30. Weinberg RS, Gould D. Fundamentos da psicologia do esporte e do exercício. Porto Alegre: Artmed; 2001.
31. Fukukawa Y, Nakashima C, Tsuboi S, Kozakai R, Doyo W, Niino N, et al. Age differences in the effect of physical activity on depressive symptoms. Psychol Aging. 2004;19(2):346-51.

32. Egito M, Matsudo S, Matsudo V. Auto-estima e satisfação com a vida de mulheres adultas praticantes de atividade física de acordo com a idade cronológica. R Bras Ci e Mov. 2005;13(3):59-66.

33. Guimarães ACA, Baptista FM. Índice de massa corporal e qualidade de vida relacionada com a saúde em mulheres de meia-idade. Rev Bras Clin Med. 2008;6:228

34. Cid L, Silva C, Alves J. Atividade física e bem-estar psicológico: perfil dos participantes no programa de exercício e saúde de Rio Maior. Motri. 2007;3(2):47-55.

35. Moriyama CK. Impacto isolado e associado da terapia hormonal e exercício físico na qualidade de vida em mulheres no climatério pós-menopausa. São Paulo. Dissertação Faculdade de Medicina da Universidade de São Paulo; 2007.

36. Dennerstein L, Smith AM, Morse C, Burger H, Green A, Hopper J, et al. Menopausal symptoms in Australian women. Med J Aust. 1993;159(4):232-6.

37. Stoppe Junior A, Louzã Neto MR. Depressão na terceira idade: apresentação clínica e abordagem terapêutica. São Paulo: Lemos; 1996.

38. Snowdon J. Qual é a prevalência de depressão na terceira idade? Rev Bras Psiquiatr. 2002;24 Suppl 1:42-7.

39. Waltrick C, Vieira ZM. Qual é a prevalência de depressão na terceira idade? Lecturas: Educación Física y Deportes. 2006;(94):31 [acesso em 17 jul 2016]; Disponível em: https://goo.gl/oYqqCs.

\section{Como citar este artigo:}

Lima JS, Matsudo SMM, Araújo TL, Matsudo VKR. Caracterização do estado de humor de mulheres fisicamente ativas e sua relação com a idade cronológica e com o índice de massa corporal. Rev. Aten. Saúde. 2016;14(49):19-26. 